\title{
The Slow Axonal Transport of the Microtubule-Associated Protein Tau and the Transport Rates of Different Isoforms and Mutants in Cultured Neurons
}

\author{
Michelle A. Utton, ${ }^{1}$ James Connell, ${ }^{1}$ Ayodeji A. Asuni, ${ }^{1}$ Marjon van Slegtenhorst, ${ }^{2}$ Michael Hutton, ${ }^{2}$ \\ Rohan de Silva, ${ }^{3}$ Andrew J. Lees, ${ }^{3}$ Chris C. J. Miller, ${ }^{1}$ and Brian H. Anderton ${ }^{1}$ \\ ${ }^{1}$ Department of Neuroscience, Institute of Psychiatry, King's College London, London SE5 8AF, United Kingdom, ${ }^{2 M a y o}$ \\ Clinic Jacksonville, Jacksonville, Florida 32224, and ${ }^{3}$ The Reta Lila Weston Institute of Neurological Studies, University \\ College London, London W1T 4JF, United Kingdom
}

\begin{abstract}
We demonstrate that the microtubule-associated protein tau, in the form of enhanced green fluorescent protein (EGFP) tau, is transported along axons of neurons in culture in the slow component of axonal transport with a speed comparable with that previously measured in vivo. It was demonstrated that the EGFP tag has no effect on transport characteristics, and the methodology enables slow transport rates of individual tau isoforms and tau mutants to be measured. We also expressed EGFP-tagged tau isoforms containing either three or four $\mathrm{C}$-terminal repeats and zero or two N-terminal inserts in cultured neurons. No significant differences were found in the
\end{abstract}

average rate of slow transport of the wild-type tau isoforms, suggesting that the exon $10 \mathrm{C}$-terminal repeat or the $\mathrm{N}$-terminal inserts do not contain regions that play a significant regulatory role in axonal transport. Similarly, we found that missense mutations in tau have no noticeable effect on the rate of transport; hence their ability to cause neurodegeneration is by another mechanism other than that affecting the overall slow axonal transport of tau.

Key words: tau; tau isoforms; tau N-terminal inserts; tau $C$-terminal repeats; slow axonal transport; neuronal cultures; transfection; EGFP; FTDP-17
The axonal transport of components synthesized in the cell body through the axon is classified as fast transport, with rates up to several hundred millimeters per day, and slow transport, moving at rates of $\sim 1 \mathrm{~mm} / \mathrm{d}$ in slow component a and several millimeters per day in slow component b (Baas and Brown, 1997; Hirokawa et al., 1997; Nixon, 1998). The axonal transport of the microtubuleassociated protein tau has been assessed by in vivo methods; reported rates vary between 0.2 and $0.4 \mathrm{~mm} / \mathrm{d}$ in retinal ganglion cells and between 1.7 and $3 \mathrm{~mm} / \mathrm{d}$ for sciatic nerve (Tytell et al., 1984; Mercken et al., 1995; Tashiro et al., 1996).

Tau in human brain consists of six isoforms, differing in the absence or presence of zero, one, or two $\mathrm{N}$-terminal inserts and either three or four C-terminal repeat regions (Francon et al., 1982; Goedert et al., 1989; Goedert and Jakes, 1990). The C terminus of tau contains the microtubule-binding regions, regulating microtubule dynamic properties (Lee and Rook, 1992; Brandt and Lee, 1993; Léger et al., 1994), and the N terminus of tau has been shown to interact with the neuronal plasma membrane (Brandt et al., 1995; Maas et al., 2000). We now have compared the rates of transport of individual tau isoforms by the direct method of monitoring the movement of enhanced green fluorescent-labeled (EGFP) tau by a previously successful technique that was used for studying the middle neurofilament subunit NF-M (Ackerley et al., 2000).

\footnotetext{
Received Nov. 20, 2001; revised May 15, 2002; accepted May 17, 2002.

This work was funded by the Alzheimer's Society, the Wellcome Trust, the Medical Research Council, the Mayo Clinic, the Progressive Supranuclear Palsy (Europe) Association, The Brain Research Trust, and the Reta Lila Weston Research Trust.

Correspondence should be addressed to Dr. Michelle Utton, Box PO37, Department of Neuroscience, Institute of Psychiatry, King's College London, De Crespigny Park, London SE5 8AF, UK. E-mail: m.utton@iop.kcl.ac.uk.

Copyright (C) 2002 Society for Neuroscience $0270-6474 / 02 / 226394-07 \$ 15.00 / 0$
}

The mechanisms of conversion of tau from a functional microtubule-associated protein to an aggregated form in many neurodegenerative diseases are still mainly unknown. Tau also is known to redistribute from its primary location in the axon to the somatodendritic compartment in disease (Götz et al., 1995). Recent reports suggest that in cultured neurons the overexpression of tau impairs the axonal transport of components such as mitochondria and neurofilaments (Stamer et al., 2002) and that fast axonal transport also may be impaired in transgenic animals overexpressing tau (Ishihara et al., 1999). It is also possible that $\mathrm{A} \beta$ may impair in vivo the axonal transport of tau itself (Götz et al., 2001).

Tau dysfunction leading to neurodegeneration has been demonstrated unequivocally with the identification of mutations in tau in frontotemporal dementia and parkinsonism linked to chromosome 17 (FTDP-17) (Hutton et al., 1998; Poorkaj et al., 1998; Spillantini et al., 1998). The mechanisms by which these mutations lead to neurodegeneration are far from clear. Some of the missense mutations have been shown to have a reduced binding to microtubules (Hasegawa et al., 1998; Hong et al., 1998; Dayanandan et al., 1999; Rizzu et al., 1999; Barghorn et al., 2000), to increase the tendency of tau to aggregate in vitro (Goedert et al., 1999; Nacharaju et al., 1999; von Bergen et al., 2001) and in vivo (Vogelsberg-Ragaglia et al., 2000), and to decrease tau degradation (Yen et al., 1999). From these studies it is clear, however, that not all of the missense mutations affect the function of tau in same manner. This suggests that as yet undiscovered mechanisms may be occurring.

To address this, we describe the first direct measurements of axonal transport of various wild-type and mutant forms of tau by directly monitoring EGFP-tagged tau that has been transfected into rat cortical neurons in culture. 


\section{MATERIALS AND METHODS}

Constructs. All tau constructs (wild type and mutants) prepared for cloning into EGFP vectors were amplified by using primers with SalI and $B g l$ II linkers for directional restriction cloning into pEGFP-C1 (EGFP fused to the $\mathrm{N}$ terminus of tau; Clontech Laboratories, Basingstoke, UK). Site-directed mutagenesis was used to introduce the tau mutations as described by Dayanandan et al. (1999). The tau mutations introduced into 0N3Rtau (0 N-terminal insert, 3 C-terminal repeat tau isoform) were V337M (valine-to-methionine mutation at position 337) and R406W (arginine-to-tryptophan mutation at position 406). The tau mutations introduced into 0N4Rtau (0 N-terminal insert, 4 C-terminal repeat tau isoform) included the following: I260V (isoleucine-to-valine mutation at position 260), delK280 (deletion of lysine residue at position 280), P301L (proline-to-leucine mutation at position 301), V337M (valine-to-methionine mutation at position 337), and R406W (arginineto-tryptophan mutation at position 406).

DNA for transfection was prepared by an endotoxin-free Maxiprep kit (Qiagen, Crawley, UK). DNA from independent maxi-preparations was used to avoid any possible batch-specific effects. 0N4Rtau also was cloned into pEGFP-N1 (Clontech Laboratories) to allow for comparison with C1 constructs. In addition, 0N4Rtau was cloned into pCIneo (Promega, Southampton, UK) to allow for comparison with the EGFP-tagged constructs.

Transfection of cortical cultures. Cortical neurons were obtained from embryonic day 18 (E18) rat embryos and cultured as described by Ackerley et al. (2000). Briefly, 6- to 7-d-old primary cortical cells were transfected by a modified calcium phosphate method with a Profection kit (Promega) as described by Ackerley et al. (2000) (essentially according to Nikolic et al., 1996; Xia et al., 1996). After incubation of the DNA/calcium phosphate precipitate on the neurons for $\sim 40 \mathrm{~min}$, the incubation was stopped by shocking with $2 \%$ (v/v) DMSO/5\% (v/v) glycerol in HEPES-buffered saline. The cells were washed three times with culture media and returned to the incubator. To determine the requirement of metabolic energy for the transport of tau, we treated cells with $50 \mathrm{~mm}$ 2-deoxy-D-glucose/0.05\% (v/v) sodium azide for $30 \mathrm{~min}$ at 120 min after transfection shock treatment (Yoon et al., 1998). Cells then were washed with culture media and further incubated to allow the cells to be fixed at given times.

Fixation of neuronal cultures and image analysis. At appropriate time points after shock the neurons were fixed with $4 \%(\mathrm{w} / \mathrm{v})$ paraformaldehyde/PBS prewarmed to $37^{\circ} \mathrm{C}$ for $20 \mathrm{~min}$ and then mounted in Vectashield (Vector Laboratories, Peterborough, UK). Neurons were examined with a Zeiss Axioskop microscope, and images were collected via a CCD camera and analyzed with MetaMorph image analysis software (Roper Scientific, Marlow, UK). The distance traveled by the tau-EGFP was measured from the perimeter of the cell body along the axon to the limit of the fluorescent front. Approximately 20-50 neurites were analyzed per time point within a data set. The average rate of tau transport was calculated from the linear regression of each experiment and averaged from between 3 and 13 independent experiments. Statistical analysis was performed with the one-way ANOVA test.

Tau-EGFP-transfected neurons also were processed by using the following fixation/extraction/permeabilization methods (protocols based on Black et al., 1996): procedure 1, as above; procedure 2, $4 \%$ (w/v) paraformaldehyde/PBS (prewarmed to $37^{\circ} \mathrm{C}$ ) for $20 \mathrm{~min}$ at room temperature, followed by incubation with $0.1 \%$ (v/v) Triton X-100/PBS for $10 \mathrm{~min}$ at room temperature; procedure $3,4 \%(\mathrm{w} / \mathrm{v})$ paraformaldehyde/ PBS (prewarmed to $37^{\circ} \mathrm{C}$ ) for $20 \mathrm{~min}$ at room temperature, followed by incubation with $0.1 \%(\mathrm{v} / \mathrm{v})$ Triton $\mathrm{X}-100 / \mathrm{PBS}$ for $2 \mathrm{~min}$ at room temperature; procedure 4, 4\% (w/v) paraformaldehyde/PBS (prewarmed to $37^{\circ} \mathrm{C}$ ) for $20 \mathrm{~min}$ at room temperature, followed by an incubation with $0.5 \%(\mathrm{v} / \mathrm{v})$ Triton X-100/PBS for $10 \mathrm{~min}$ at room temperature; procedure 5 (fixation without extraction), $0.3 \%$ (v/v) glutaraldehyde/PEM (80 mM PIPES, 5 mM EGTA, 1 mM magnesium chloride, $\mathrm{pH} 6.8$; prewarmed to $37^{\circ} \mathrm{C}$ ) for $20 \mathrm{~min}$ at room temperature, followed by permeabilization with $0.5 \%(\mathrm{v} / \mathrm{v})$ Triton X-100/PBS for $10 \mathrm{~min}$ at room temperature; procedure 6 (combined fixation/extraction), $0.3 \%$ (v/v) glutaraldehyde/PEM $/ 0.5 \%$ (v/v) NP-40 (prewarmed to $37^{\circ} \mathrm{C}$ ) for $10 \mathrm{~min}$ at room temperature. All fixation procedures that used glutaraldehyde were subjected to the following incubations after fixation/permeabilization: $10 \mathrm{mg} / \mathrm{ml}$ sodium borohydride in PBS for $7 \mathrm{~min}$, rinsed with PBS, $0.1 \mathrm{M}$ glycine in PBS for $20 \mathrm{~min}$, rinsed with PBS, and incubation with $1 \%(\mathrm{w} / \mathrm{v})$ blocking solution (BSA/PBS) for $30 \mathrm{~min}$. All incubations were performed at room temperature.
Nocodazole treatment of transfected neurons and immunofluorescence. At $24 \mathrm{hr}$ after transfection the neurons were treated with $5 \mu \mathrm{g} / \mathrm{ml}$ nocodazole (Tocris, Bristol, UK) for $30 \mathrm{~min}$ at $37^{\circ} \mathrm{C}$. Neurons then were fixed by using the combined fixation/extraction procedure 6 as detailed above. Untreated and treated neurons were stained for tubulin with the DM1A antibody (Sigma, Poole, UK), followed by incubation with a Texas Red-labeled secondary antibody (Vector Laboratories).

Analysis of tau extracted from neurons. To assess the removal of soluble tau released from neurons when the combined fixation/extraction procedure 6 was used, we treated neurons with either PBS for $20 \mathrm{~min}$ or $0.5 \%$ (v/v) NP-40/PEM for $10 \mathrm{~min}$ (to mimic the extraction conditions). The solution was removed from the neurons, and total protein was precipitated by the addition of $\sim 8$ volumes of cold methanol (Black et al., 1996). The resulting methanol-precipitated material was collected by centrifugation, air-dried, and dissolved in SDS-PAGE sample buffer (Sambrook et al., 1989). The neurons were scraped directly into SDS-PAGE sample buffer. The resulting material was analyzed by SDS-PAGE and immunoblotting with the polyclonal tau antibody TP70 (Brion et al., 1993).

Immunofluorescence microscopy. Costaining of tau-EGFP-transfected neurons for the dendritic marker MAP2 was performed by using MAP2a/b monoclonal antibody (Sigma), followed by incubation with a Texas Red-labeled secondary antibody (Vector Laboratories). Neurons that were transfected with 0N4Rtau cloned into pCIneo were stained with a four-repeat tau-specific antibody raised to the additional repeat region present in 4Rtau [antibody raised against peptide IINKKLDLSNVQSK, corresponding to amino acids 277-290; numbering according to htau40 (2N4Rtau, 441 amino acids) (Goedert et al., 1989)]. The antibody was used at a concentration of 1:200 for $2 \mathrm{hr}$ at room temperature, followed by incubation with a FITC-labeled secondary antibody (Vector Laboratories).

\section{RESULTS}

\section{Direct visualization of tau transport along axons}

We have analyzed the rate of anterograde transport of tau by transfecting rat cortical neuronal cultures with tau-EGFP (Nterminally tagged) and analyzing the cells by fluorescence microscopy after fixation up to $300 \mathrm{~min}$ after transfection. Cells were analyzed randomly by measuring the distance traveled by the tau-EGFP from the perimeter of the cell body to the boundary of the fluorescent front along the longest neurite (also see Ackerley et al., 2000). Representative experiments were analyzed by independent workers, and no significant difference in the distance to the fluorescent front was observed, demonstrating that the limit of the fluorescent front was identified easily. Measurements were taken up to $300 \mu \mathrm{m}$ from the cell body to avoid including neurites in which tau-EGFP had reached the neurite terminals. Previous studies have demonstrated that the average length of neurites of cortical neurons under these experimental conditions exceeded $700 \mu \mathrm{m}$; hence the assays are of transport within the neurites and are not a reflection of neurite outgrowth rates (Ackerley et al., 2000). Figure 1 shows representative images of 0N4Rtau-EGFP transported along the neurites; $a-d$ show examples of neurites taken at 120, 180, 210, and $240 \mathrm{~min}$ after transfection shock treatment, showing an approximate travel distance from the cell body of 50, 100, 120, and $150 \mu \mathrm{m}$, respectively. EGFP when transfected alone (data not shown) (Ackerley et al., 2000) was observed along the length of all processes at early time points, indicating a possible movement by fast axonal transport.

To analyze whether the tau fusion proteins were sorted correctly to axons after transient transfection, we costained 0N4Rtau-EGFP-transfected neurons for endogenous MAP2, a microtubule-associated protein localized to dendrites. Analysis shows that 0N4Rtau-EGFP protein was sorted to the axons (Fig. 2). In addition, 0N4Rtau-EGFP was found to be present in the cell body and dendrites, although this is not unusual because a variety of exogenously expressed axonal proteins has been shown to exhibit this distribution either because of saturation of the 


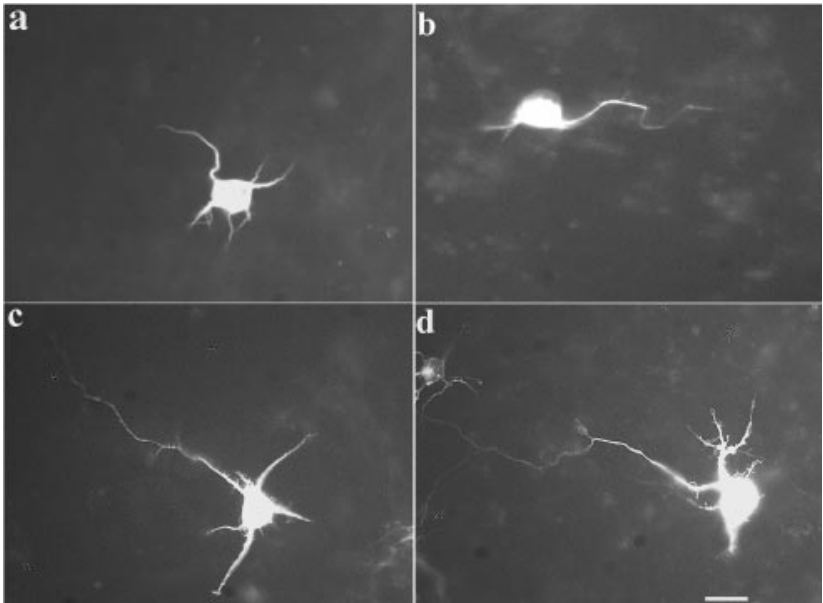

Figure 1. Slow axonal transport of 0N4Rtau-EGFP in transfected cortical neurons. The 6- to 7-d-old neurons were transfected with 0N4RtauEGFP. Representative images are shown of the neurons: $a, 120 \mathrm{~min} ; b$, $180 \mathrm{~min} ; c$, $210 \mathrm{~min}$; $d, 240 \mathrm{~min}$ after transfection. Scale bar, $20 \mu \mathrm{m}$.

\section{Tau-GFP}

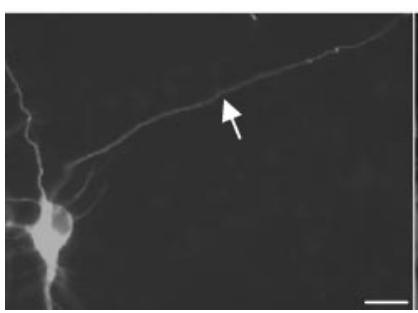

Figure 2. EGFP-tagged tau is sorted to axons. 0N4Rtau-EGFPtransfected neurons were fixed after $1 \mathrm{~d}$ and processed for immunofluorescence with the anti-MAP2 antibody. Arrows indicate axon-expressing 0N4Rtau-EGFP as defined by the absence of MAP2 labeling. Scale bar, $20 \mu \mathrm{m}$.

axonal sorting machinery (for review, see Winckler and Mellman, 1999) or because neuronal process selectivity mechanisms downstream of transport occur at the plasma membrane as suggested for NgCAM-GFP (Burack et al., 2000). Although 0N4RtauEGFP is present in the dendrites, showing the usual thicker, more tapered process morphology compared with axons, it is clear that the 0N4Rtau-EGFP present in the axons is clearly identifiable, given the thinner uniform morphology of the process and the lack of staining for MAP2. This method is therefore a legitimate neuronal model in which to study the axonal transport of tauEGFP, and all of the measurements of axonal transport of tau were made on the longest and thinnest process because this is most likely the axon of the studied cells.

\section{Axonal transport of tau is an energy-dependent process}

To assess the requirement for metabolic energy for the transport of tau, we incubated cells with 2-deoxy-D-glucose/sodium azide, which is known to inhibit energy-dependent metabolic processes (Yoon et al., 1998) (Fig. 3). The transport of 0N4Rtau-EGFP was halted after the deoxyglucose treatment; after the inhibitors were washed out, the transport rate resumed at a rate comparable with that of untreated cells, showing that the cessation of tau transport because of the inhibition of metabolic processes was reversible on removal of the treatment and that no subsequent detrimental effect on tau transport was observed.

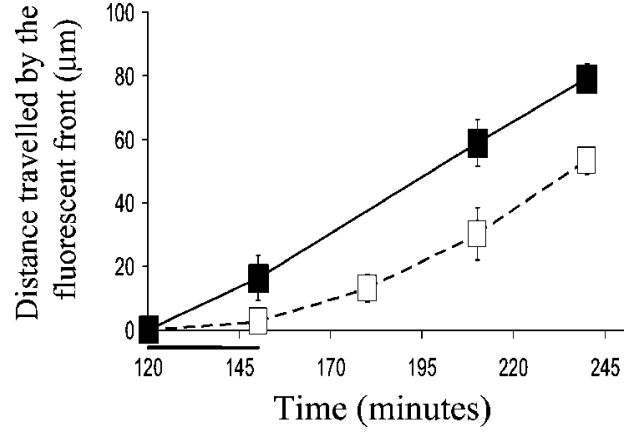

Figure 3. Axonal transport of tau is energy dependent. The distance traveled by the 0N4Rtau-EGFP species, from the cell body to the fluorescent front, is shown over time. Neurons were treated with 2-deoxy-Dglucose/sodium azide for $30 \mathrm{~min}$ at a point $120 \mathrm{~min}$ after transfection (shown by the horizontal bar). Then the neurons were washed, incubated in culture medium, and fixed at appropriate time intervals after transfection. Shown are 0N4Rtau-EGFP-transfected neurons (untreated; filled squares) and 0N4Rtau-EGFP-transfected neurons treated with deoxy-Dglucose/sodium azide (open squares). Error bars indicate SEM; one-way ANOVA showed significant differences between untreated and treated neurons at the 210 and 240 time points $(p=0.04$ and $p=0.002$, respectively).

The average distance traveled by tau-EGFP at the first time point that was analyzed differed by $\sim 0-20 \mu \mathrm{m}$ between experiments. These differences are attributed to the small variations in the time of expression of the recombinant protein after transfection (Ackerley et al., 2000). To facilitate comparisons among the experimental data shown in Figures 3, 4, 6, and 7, we have depicted the distance traveled as the distance from the first measurement, which is recorded as zero.

\section{Incorporation or position of the EGFP tag on the tau molecule does not affect the transport of tau}

We investigated whether the inclusion or position of the EGFP tag affected the rate of transport of transfected tau. The transport of untagged 0N4Rtau expressed in pCIneo was compared with that of 0N4Rtau-EGFP (Fig. 4). Figure $4 A$ shows representative images of untagged 0N4Rtau transported along the neurites. Neurons that were transfected with 0N4Rtau-pCIneo were fixed and stained with a 4Rtau-specific antibody and were analyzed in an identical way to those transfected with 0N4Rtau-EGFP (Fig. $4 B)$. The overall slow rate of axonal transport of untagged 0N4Rtau was $0.873 \pm 0.005 \mathrm{~mm} / \mathrm{d}$ compared with $0.916 \pm 0.035$ $\mathrm{mm} / \mathrm{d}$ for 0N4Rtau-EGFP (not significantly different with oneway ANOVA test; $p=0.3$ ).

The overall rate of tau transport was not affected by the position of the EGFP tag because the average rate of transport of 0N4Rtau-EGFP expressed in pEGFP-N1 (tau tagged with GFP at its $\mathrm{C}$ terminus) was comparable with that expressed in pEGFP$\mathrm{C} 1$, i.e., $\mathrm{N}$-terminally tagged $[\mathrm{N} 1=1.030 \pm 0.028 \mathrm{~mm} / \mathrm{d}$ (data not shown); $\mathrm{C} 1=1.003 \pm 0.093 \mathrm{~mm} / \mathrm{d}$ (not significantly different with one-way ANOVA test; $p=0.8)$ ].

\section{Axonal tau-EGFP is present as both a microtubule- bound and a soluble fraction}

Because tau exists in neurons in at least two compartments, microtubule-bound and soluble tau, it was important to determine whether bound and soluble tau might be transported at different rates. This was investigated by making measurements of neurons fixed in ways so that these two different compartments might be distinguished. Thus a range of fixation/extraction/permeabiliza- 
A.

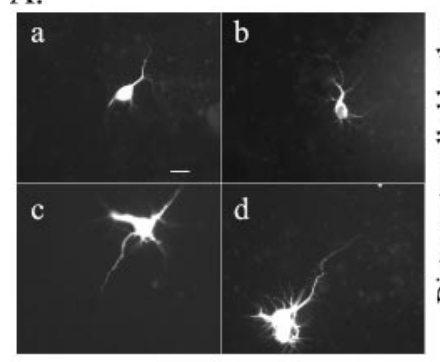

B.

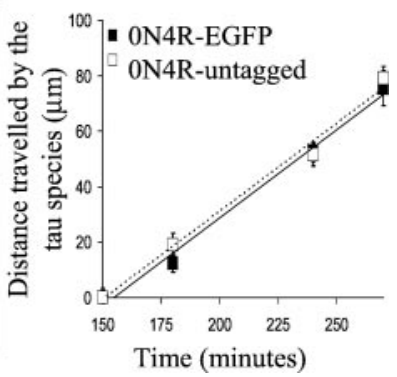

Figure 4. Inclusion of the EGFP tag does not interfere with the axonal transport of tau. A, Slow axonal transport of untagged 0N4Rtau in transfected cortical neurons. The 6- to 7-d-old neurons were transfected with 0N4Rtau-pCIneo and processed for immunofluorescence with a 4Rtau-specific antibody, followed by incubation with a FITC-labeled secondary antibody. Representative images are shown of the neurons: $a$, $150 \mathrm{~min} ; b, 180 \mathrm{~min} ; c, 240 \mathrm{~min} ; d, 270 \mathrm{~min}$ after transfection. Scale bar, $20 \mu \mathrm{m}$. B , Comparison of axonal transport of 0N4Rtau-EGFP ( filled squares) and untagged 0N4Rtau (open squares). The distance traveled by the tau species, from the cell body to the fluorescent front, is shown over time. Per experiment, 20-50 neurons were measured for each time point; the distance traveled was pooled from three independent experiments. Error bars indicate SEM; one-way ANOVA revealed no significant differences between overall axonal transport rate of the 0N4Rtau-EGFP and the untagged 0N4Rtau (obtained by averaging the rates from each experimental data set as determined by linear regression; $p=0.3$ ).

tion procedures was compared, and the distances traveled by 0N4Rtau-EGFP $310 \mathrm{~min}$ after transfection were determined. With procedure 1 involving fixation only with PFA, the average distance traveled by 0N4Rtau-EGFP was $197.2 \mu \mathrm{m}( \pm 11.2)$. With the use of procedures 2, 3, and 4 involving fixation with PFA followed by permeabilization with $0.1 \%$ (v/v) Triton X-100 for 2 or $10 \mathrm{~min}$ and $0.5 \%(\mathrm{v} / \mathrm{v})$ Triton X-100 for $10 \mathrm{~min}$, respectively, the average distance traveled was $203.67 \mu \mathrm{m}$ ( \pm 21.2$), 207.53 \mu \mathrm{m}$ $( \pm 12.4)$, and $194.23 \mu \mathrm{m}( \pm 15.2)$. With the use of procedure 5 involving fixation with glutaraldehyde without extraction followed by permeabilization with Triton X-100, the average distance traveled was $194.75 \mu \mathrm{m}( \pm 14.8)$. With the use of procedure 6 involving a combined fixation/extraction step, the average distance traveled was $197.14 \mu \mathrm{m}( \pm 8.1)$. Fixation methods followed by permeabilization (procedures 2, 3, 4, and 5) did not alter the measured distance that 0N4Rtau-EGFP traveled as assessed after fixation alone. When unbound tau was extracted (procedure 6), the measured distance traveled by the tau-EGFP was statistically no different from when unbound tau was still present. This indicates in our experimental system that a portion of tau-EGFP within the axon is microtubule bound but that unbound tau apparently is not transported at a faster rate than that associated with microtubules.

It was important to verify that the fixation/extraction procedure 6 indeed did behave as reported by Black et al. (1996) under our experimental conditions. Therefore, 0N4Rtau-EGFPtransfected neurons either were fixed by procedure 1 or fixed/ extracted by procedure 6 and then processed by immunofluorescence for tubulin staining ( $24 \mathrm{hr}$ after transfection). Figure $5 a$ shows EGFP-tagged tau labeling of a neuron fixed by procedure 1 (Fig. $5 b$ shows the corresponding tubulin stain). The diff use fluorescence of tau-EGFP in Figure 5 is likely to be attributable to the presence of both cytoplasmic and microtubule-bound tau. By contrast, after fixation/extraction by procedure 6 the tauEGFP appears to colocalize with microtubules (Fig. $5 c, d$ ), shown by the sharp filamentous tau-EGFP fluorescence within some of

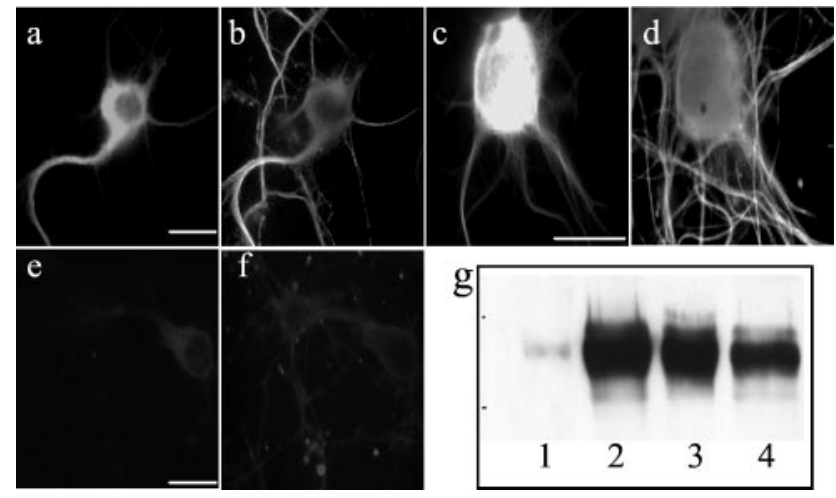

Figure 5. Analysis of neurons processed by a combined fixation/extraction procedure. $a, b, 0 \mathrm{~N} 4 \mathrm{R}-\mathrm{EGFP}$-transfected neurons processed via procedure 1 (see Materials and Methods); $a$ shows 0N4R-EGFP, and $b$ shows the corresponding tubulin staining. $c, d$, 0N4R-EGFP-transfected neurons processed via fixation/extraction procedure 6 ; $c$ shows $0 \mathrm{~N} 4 \mathrm{R}$ $\mathrm{EGFP}$, and $d$ shows the corresponding tubulin staining. $e, f, 0 \mathrm{~N} 4 \mathrm{R}-\mathrm{EGFP}$ transfected neurons treated with $5 \mu \mathrm{g} / \mathrm{ml}$ nocodazole for $30 \mathrm{~min}$ and processed via fixation/extraction procedure $6 ; e$ shows 0 N4R-EGFP, and $f$ shows the corresponding tubulin staining. Scale bar, $20 \mu \mathrm{m}$. $g$, Immunoblotting for tau in neuronal cell lysates and extracted proteins. Lane 1, Extracted tau after incubation with PBS for $20 \mathrm{~min}$ (as in procedure 1); lane 2, cell lysate after incubation with PBS for $20 \mathrm{~min}$; lane 3, extracted tau after incubation with $0.5 \%(\mathrm{v} / \mathrm{v}) \mathrm{NP}-40$ for $10 \mathrm{~min}$ (as in procedure 6); lane 4, cell lysate after incubation with $0.5 \%$ (v/v) NP-40 for $10 \mathrm{~min}$. Molecular weight markers on the left correspond to $39.8 \mathrm{kDa}$ (bottom marker) and $58.1 \mathrm{kDa}$ (top marker).

the neuronal processes. The sharper tau-EGFP fluorescence in neurons processed by procedure 6 is evident probably because the cytoplasmic tau was removed, as shown by Black et al. (1996). To confirm this interpretation, we treated neurons with nocodazole, a microtubule-depolymerizing drug, and then processed them by the fixation/extraction procedure 6 (Fig. 5e,f). Tau-EGFP fluorescence and tubulin immunofluorescence virtually were abolished, indicating that after microtubule depolymerization fixation/extraction procedure 6 removed most of the tau and tubulin. Thus under conditions in which microtubules remained assembled, only unbound cytoplasmic tau was extracted (Fig. $5 c, d$ ). This was confirmed biochemically; neurons were treated with or without the extraction component of procedure $6[0.5 \%(\mathrm{v} / \mathrm{v})$ NP-40], and any extracted protein was precipitated and analyzed by immunoblotting for tau (Fig. $5 g$ ). Without the extraction detergent nearly all tau protein remained within the cell lysate, as expected (Fig. 5g, lanes 1, 2). However, after incubation with the extraction detergent a considerable proportion of tau was extracted into the solution (Fig. 5g, lanes 3, 4). (Immunoblotting was repeated as above with cells processed with the complete fixation/ extraction solution; because of the smeary nature of the gels caused by the analysis of fixed cells by SDS-PAGE, these data are not shown, although the profile of the tau extracted from the cells was the same as that shown in Fig. $5 g$ ). These results confirm that fixation/extraction procedure 6 does remove cytoplasmic tau, with the unextracted tau being colocalized, i.e., bound, to the microtubules (Fig. 5c,d).

\section{Presence of $\mathbf{N}$-terminal inserts and the exon 10 C-terminal repeat have no significant effect on the rate of tau transport}

We next compared the overall slow rate of anterograde axonal transport of the following wild-type tau isoforms: 0N3R, 0N4R, 2N3R (2 N-terminal insert, $3 \mathrm{C}$-terminal repeat tau isoform), and 


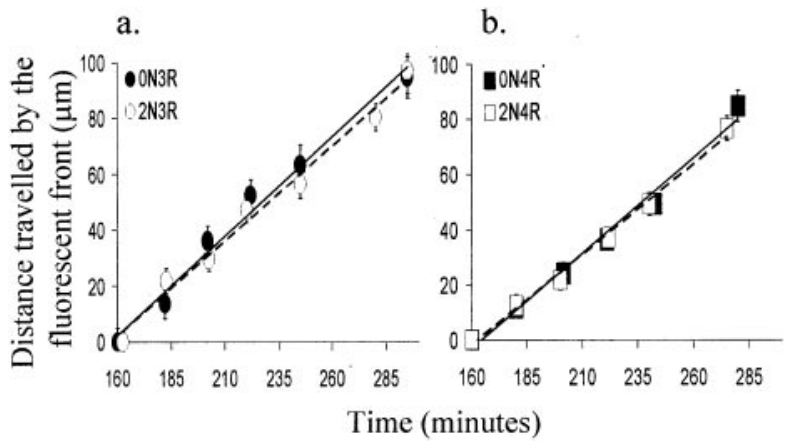

Figure 6. Overall slow axonal transport of tau isoforms. $a$, Comparison of axonal transport of $0 \mathrm{~N} 3 \mathrm{R}$ and $2 \mathrm{~N} 3 \mathrm{R}$ tau-EGFP. $b$, Comparison of axonal transport of 0 N4R and 2N4Rtau-EGFP. The distance traveled by the tau-EGFP species, from the cell body to the fluorescent front, is shown over time. Per experiment, 20-50 neurons were measured for each time point; the distance traveled was pooled from three to five independent experiments. Error bars indicate SEM; one-way ANOVA revealed no significant differences among overall axonal transport rates of the wild-type tau isoforms (obtained by averaging the rates from each experimental data set as determined by linear regression; $p>0.8$ ).

2N4Rtau (2 N-terminal insert, $4 \mathrm{C}$-terminal repeat tau isoform) all tagged with EGFP (Fig. 6). The data shown are from three to five independent experiments; error bars indicate SEM. Within each experiment 20-50 neurites were measured for each data point. The overall slow rate of axonal tau transport was determined by averaging the rate generated by linear regression for each experimental data set. When we compared different isoforms of tau, the overall rate of transport for 0N3Rtau-EGFP was $0.965 \pm 0.157 \mathrm{~mm} / \mathrm{d}$; for $2 \mathrm{~N} 3 \mathrm{R}$ tau-EGFP it was $0.931 \pm 0.071$ $\mathrm{mm} / \mathrm{d}$; for $0 \mathrm{~N} 4 \mathrm{Rtau}-\mathrm{EGFP}$ it was $0.952 \pm 0.064 \mathrm{~mm} / \mathrm{d}$; for 2 N4Rtau-EGFP it was $0.927 \pm 0.082 \mathrm{~mm} / \mathrm{d}$. The axonal transport rate of tau determined by this method was comparable with that classically known as the slow transport component as measured in vivo. No statistically significant differences in the overall slow rate of axonal transport of tau containing either zero or two $\mathrm{N}$-terminal inserts or three or four $\mathrm{C}$-terminal repeats were seen (not significantly different with one-way ANOVA test; $p>0.4$ for comparisons of $0 \mathrm{~N} 3 \mathrm{R}$ to $0 \mathrm{~N} 4 \mathrm{R}, 0 \mathrm{~N} 3 \mathrm{R}$ to $2 \mathrm{~N} 3 \mathrm{R}$, and $0 \mathrm{~N} 4 \mathrm{R}$ to 2N4R).

\section{Mutations found in FTDP-17 have no observable effect on the rate of tau transport}

We compared the overall rate of axonal transport of EGFPtagged wild-type tau and a variety of mutant EGFP-tagged tau species found in FTDP-17. Figure $7 a-c$ shows a comparison among wild-type 0N3Rtau-EGFP, 0N4Rtau-EGFP, and the tauEGFP species bearing the following FTDP-17 mutations: 0N3Rtau-V337M, 0N3Rtau-R406W, 0N4Rtau-I260V, 0N4RtaudelK280, 0N4Rtau-P301L, 0N4Rtau-V337M, and 0N4RtauR406W. Figure $7 d$ shows the overall slow transport rates of all of the tau species that were analyzed. The average rate of tau transport for 0N3Rtau was $1.128 \pm 0.084 \mathrm{~mm} / \mathrm{d}$; 0N3R-V337M was $1.154 \pm 0.095 \mathrm{~mm} / \mathrm{d} ; 0 \mathrm{~N} 3 \mathrm{R}-\mathrm{R} 406 \mathrm{~W}$ was $1.111 \pm 0.131 \mathrm{~mm} / \mathrm{d}$; 0N4Rtau was $1.003 \pm 0.093 \mathrm{~mm} / \mathrm{d}$; 0N4R-I260V was $1.138 \pm$ $0.113 \mathrm{~mm} / \mathrm{d}$; 0N4R-delK280 was $1.192 \pm 0.122 \mathrm{~mm} / \mathrm{d}$; 0N4R$\mathrm{P} 301 \mathrm{~L}$ was $1.133 \pm 0.107 \mathrm{~mm} / \mathrm{d} ; 0 \mathrm{~N} 4 \mathrm{R}-\mathrm{V} 337 \mathrm{M}$ was $1.174 \pm 0.067$ $\mathrm{mm} / \mathrm{d}$; 0N4R-R406W was $1.026 \pm 0.085 \mathrm{~mm} / \mathrm{d}$ (independent experiment number, $n,=4-10)$. No significant differences in the overall slow rate of axonal transport of tau, either wild-type or tau bearing FTDP-17 mutations, were seen (not significantly different
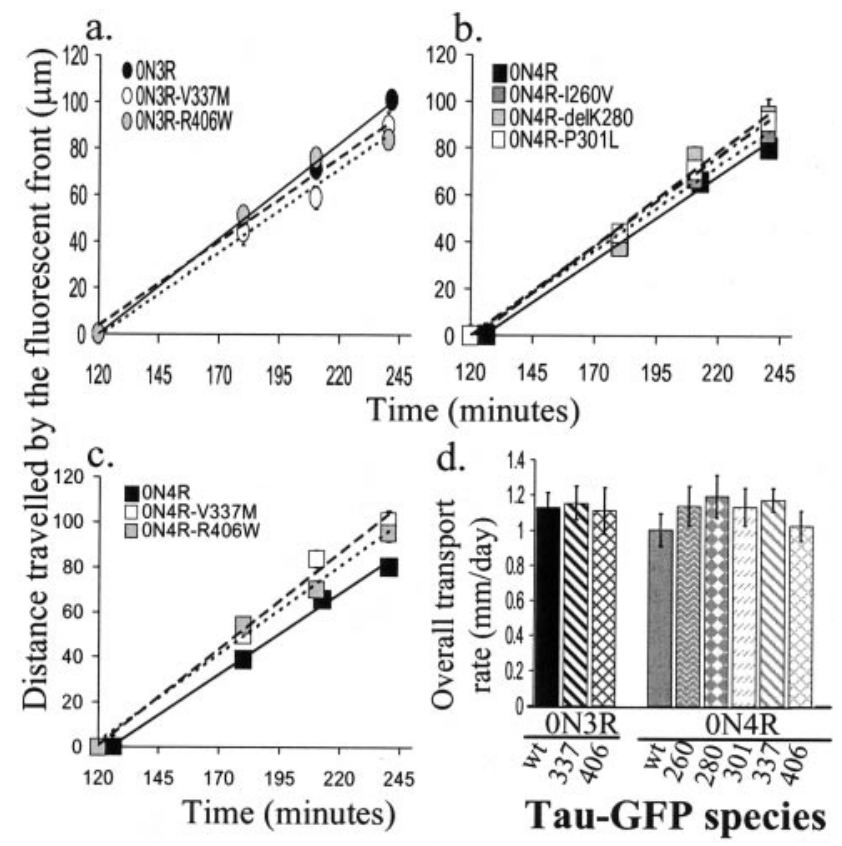

Figure 7. Overall slow axonal transport of wild-type tau isoforms and tau containing FTDP-17 mutations. $a$, Comparison of the transport of 0N3Rtau-EGFP, 0N3Rtau-V337M-EGFP, and 0N3Rtau-R406W-EGFP mutants. $b$, Comparison of the transport of 0N4Rtau, 0N4Rtau-I260VEGFP, 0N4Rtau-delK280-EGFP, and 0N4Rtau-P301L-EGFP mutants. $c$, Comparison of the transport of 0N4Rtau, 0N4Rtau-V337M-EGFP, and 0N4Rtau-R406W-EGFP mutants. The distance traveled by the tau-EGFP species, from the cell body to the fluorescent front, is shown over time. Per experiment, 20-50 neurons were measured for each time point; the distance traveled was pooled from 4 to 10 independent experiments. Error bars indicate SEM. $d$, Summary of the average overall axonal transport rates of wild-type and mutant forms of tau. Rates were calculated from a linear regression of each experimental data set and presented as the distance traveled in millimeters per day. Error bars indicate SEM. The average rate of tau transport for 0N3Rtau-EGFP was $1.128 \pm 0.084$ $\mathrm{mm} / \mathrm{d}$; 0N3Rtau-V337M-EGFP was $1.154 \pm 0.095 \mathrm{~mm} / \mathrm{d} ; 0 \mathrm{~N} 3 \mathrm{Rtau}-$ R406W-EGFP was $1.111 \pm 0.131 \mathrm{~mm} / \mathrm{d}$; 0N4Rtau-EGFP was $1.003 \pm$ $0.093 \mathrm{~mm} / \mathrm{d}$; 0N4Rtau-I260V-EGFP was $1.138 \pm 0.113 \mathrm{~mm} / \mathrm{d}$; 0N4RtaudelK280-EGFP was $1.192 \pm 0.122 \mathrm{~mm} / \mathrm{d} ; 0$ ON4Rtau-P301L-EGFP was $1.133 \pm 0.107 \mathrm{~mm} / \mathrm{d} ; 0 \mathrm{~N} 4 \mathrm{Rtau}-\mathrm{V} 337 \mathrm{M}-\mathrm{EGFP}$ was $1.174 \pm 0.067 \mathrm{~mm} / \mathrm{d}$; 0N4Rtau-R406W-EGFP was $1.026 \pm 0.085 \mathrm{~mm} / \mathrm{d}$. One-way ANOVA revealed no significant differences in the overall axonal transport rates between the wild-type tau isoforms and the mutant species (all comparisons of transport rates of wild-type tau and mutant tau; $p>0.3$ ).

with one-way ANOVA test; $p>0.3$ for comparisons of wild-type tau to mutated tau).

\section{DISCUSSION}

Aberrant deposition of tau in neurons, much of it perikaryal, typifies the tauopathies, but the mechanisms underlying this deposition are unknown. One possible mechanism is the disruption of axonal transport such that tau would accumulate in the cell body. After injection with $A \beta_{42}$ fibrils, enhanced tau pathology was observed in transgenic mice expressing mutant tau (Götz et al., 2001). Given the anatomical separation of amyloid deposition and tangle formation, it was suggested that amyloid fibrils impaired the axonal transport of tau (Götz et al., 2001). In other studies overexpression of the smallest isoform of tau in transgenic mice resulted in tau inclusions and reduced fast axonal transport of organelles (Ishihara et al., 1999), and overexpression of tau in both neuronal and non-neuronal cells also reduced kinesindependent fast transport of vesicles, neurofilaments, and mito- 
chondria (Ebneth et al., 1998; Trinczek et al., 1999; Stamer et al., 2002). Disruption of axonal transport in neurodegenerative disease is therefore a mechanism worthy of further exploration, particularly because the slowing of neurofilament transport is an early pathological feature of amyotrophic lateral sclerosis (ALS) (Collard et al., 1995; Zhang et al., 1997; Williamson and Cleveland, 1999) and mutated kinesin isoforms have been shown to underlie human peripheral neuropathy (Zhao et al., 2001). Overexpression of APPL, the Drosophila homolog of APP, also has been shown to disrupt axonal transport (Torroja et al., 1999). Based on studies of transgenic mice and Drosophila, it also has been suggested that disturbed axonal transport of amyloid precursor protein (APP) and apolipoprotein E contribute to neuronal death in these models and hence in Alzheimer's disease (Tesseur et al., 2000; Gunawardena and Goldstein, 2001).

Brain tau exists as six alternatively spliced isoforms; to date, five splice site mutations, 14 missense mutations, and a $3 \mathrm{bp}$ deletion have been identified in $>50$ FTDP-17 families (for review, see Goedert et al., 1998; van Slegtenhorst et al., 2000). The splice site mutations lead to an overproduction of four-repeat tau, implying the importance of maintaining tight ratios of threeto four-repeat tau for a functional system (Hutton et al., 1998; Spillantini et al., 1998; Varini et al., 1999). We therefore set out to investigate the transport characteristics of different tau isoforms and certain mutants in a cultured neuronal system developed in our laboratory (Ackerley et al., 2000). This system has certain advantages over animal studies and is more versatile. Individual tau species, including mutants, can be followed in the presence of endogenous tau by appropriate tagging, and the experiments can be conducted quickly and efficiently, unlike time-consuming in vivo transport studies that would require numerous transgenic animals to be generated.

Using this experimental system, we have demonstrated that the EGFP-tagged tau is localized in axons, although it is also present in dendrites. Our data on comparing different fixation/extraction protocols demonstrate that a portion of the tau-EGFP expressed within the axons is microtubule bound and that soluble tau apparently was not transported at a faster rate than microtubulebound tau. Unlike Aronov et al. (2001), we did not confirm the requirement of a 3 '-untranslated region for axonal targeting and conclude that this may be restricted to certain neuronal cell types, because they studied differentiated P19 embryonic carcinoma cells. We found that the presence of an EGFP tag had no effect on the slow transport rate of tau; hence these constructs do seem to be valid tools for studying tau transport. The slow transport of tau was shown to be energy-dependent, as we had shown previously was the case for slow transport of neurofilaments and $\alpha$-synuclein (Ackerley et al., 2000; A. Saha, M. Utton, J. Hill, R. Dayanandan, A. Asuni, S. Ackerley, A. Grierson, C. Miller, A. Davies, S. Lovestone, V. Buchman, B. Anderton, and D. Hanger, unpublished observations). The overall anterograde transport of tau was found by this method to be $\sim 1 \mathrm{~mm} / \mathrm{d}$, which corresponds with the reported rate for the slow component a of axonal transport. This rate differs from those initially reported in the literature, measuring in vivo transport of tau by radioisotopic labeling, of $0.2-0.4$ $\mathrm{mm} / \mathrm{d}$ in retinal ganglion cells. It is well known that variation in the transport rate of proteins has been observed between different cell types (Galbraith and Gallant, 2000), and, given the different methodology used here and the fact that tau transport in cortical neurons has not been reported, it is not unexpected to observe different overall rates of transport from those previously reported in vivo.
Functional differences between 3Rtau and 4Rtau isoforms have been demonstrated in a number of studies both in vitro and in cells. For example, 4Rtau binds to microtubules with higher affinity than 3Rtau (Butner and Kirschner, 1991; Gustke et al., 1994; Ackmann et al., 2000), and the inter-repeat regions of 3R and 4Rtau may act in part by distinct molecular mechanisms (Goode et al., 2000). It also appears that 3Rtau may be more susceptible to oxidative stress both in vitro and in cells, again highlighting functional differences between the isoforms under certain conditions (Utton et al., 2001). So far, however, no functional differences between tau isoforms with $0 \mathrm{~N}, 1 \mathrm{~N}$, or $2 \mathrm{~N}$ terminal inserts have been described. We therefore investigated whether variation in either the number of $\mathrm{C}$-terminal repeats or $\mathrm{N}$-terminal inserts and the mutants of tau might affect tau transport.

Our results show that none of these intrinsic variants of tau have any effect on tau transport, which is an important observation because reduced transport from the cell body could account for the accumulation of perikaryal tau and hence inclusion body formation in the tauopathies. In the case of tau it is more likely, therefore, that excesses of either 4Rtau or 3Rtau in FTDP-17 and Pick's disease or mutant forms of tau affect other properties such as differences in microtubule dynamic properties (for review, see Lee et al., 2001). However, Götz et al. (2001) have suggested that extracellular $\mathrm{A} \beta_{42}$ fibrils may cause a reduction in axonal transport because the presence of tangles in a transgenic mouse with mutant tau is anatomically remote from the $\mathrm{A} \beta$ deposits. Axonal transport of tau therefore still may be important in the tauopathies because an imbalance of tau isoforms or the presence of mutant taus may render tau more susceptible to extraneous factors that reduce transport rates; these could include $\mathrm{A} \beta$ deposits and oxidative stress. The experimental system that we have described herein is therefore likely to prove to be a powerful new tool to investigate putative pathogenic mechanisms for these diseases.

\section{REFERENCES}

Ackerley S, Grierson AJ, Brownlees J, Thornhill P, Anderton BH, Leigh PN, Shaw CE, Miller CCJ (2000) Glutamate slows axonal transport of neurofilaments in transfected neurons. J Cell Biol 150:1-11.

Ackmann M, Wiech H, Mandelkow E (2000) Nonsaturable binding indicates clustering of tau on the microtubule surface in a paired helical filament-like conformation. J Biol Chem 275:30335-30343.

Aronov S, Aranda G, Behar L, Ginzburg I (2001) Axonal tau mRNA localization coincides with tau protein in living neuronal cells and depends on axonal targeting signal. J Neurosci 21:6577-6587.

Baas PW, Brown A (1997) Slow axonal transport: a polymer transport model. Trends Cell Biol 7:380-384.

Barghorn S, Zheng-Fischhöfer Q, Ackmann M, Biernat J, von Bergen M, Mandelkow EM, Mandelkow E (2000) Structure, microtubule interactions, and paired helical filament aggregation by tau mutants of frontotemporal dementia. Biochemistry 39:11714-11721.

Black MM, Slaughter T, Moshiach S, Obrocka M, Fischer I (1996) Tau is enriched on dynamic microtubules in the distal region of growing axons. J Neurosci 16:3601-3619.

Brandt R, Lee G (1993) Functional organization of microtubuleassociated protein tau. Identification of regions which affect microtubule growth, nucleation, and bundle formation in vitro. J Biol Chem 268:3414-3419.

Brandt R, Léger J, Lee G (1995) Interaction of tau with the neural plasma membrane mediated by tau's amino-terminal projection domain. J Cell Biol 131:1327-1340.

Brion J-P, Couck A-M, Robertson J, Loviny TLF, Anderton BH (1993) Neurofilament monoclonal antibodies RT97 and 8D8 recognize different modified epitopes in paired helical filament-tau in Alzheimer's disease. J Neurochem 60:1372-1382.

Burack MA, Silverman MA, Banker G (2000) The role of selective transport in neuronal protein sorting. Neuron 26:465-472.

Butner KA, Kirschner MW (1991) Tau protein binds to microtubules through a flexible array of distributed weak sites. J Cell Biol 115:717-730. 
Collard J-F, Cole F, Julien J-P (1995) Defective axonal transport in a transgenic mouse model of amyotrophic lateral sclerosis. Nature 375:61-64.

Dayanandan R, van Slegtenhorst M, Mack T, Ko L, Yen S-H, Leroy K, Brion J-P, Anderton BH, Hutton M, Lovestone S (1999) Mutations in tau reduce its microtubule-binding properties in intact cells and affect its phosphorylation. FEBS Lett 446:228-232.

Ebneth A, Godemann R, Stamer K, Illenberger S, Trinczek B, Mandelkow EM, Mandelkow E (1998) Overexpression of tau protein inhibits kinesin-dependent trafficking of vesicles, mitochondria, and endoplasmic reticulum: implications for Alzheimer's disease. J Cell Biol 143:777-794.

Francon J, Lennon AM, Fellous A, Mareck A, Pierre M, Nunez J (1982) Heterogeneity of microtubule-associated proteins and brain development. Eur J Biochem 129:465-471.

Galbraith JA, Gallant PE (2000) Axonal transport of tubulin and actin. J Neurocytol 29:889-911.

Goedert M, Jakes R (1990) Expression of separate isoforms of human tau protein: correlation with the tau pattern in brain and effects on tubulin polymerization. EMBO J 9:4225-4230.

Goedert M, Spillantini MG, Jakes R, Rutherford D, Crowther RA (1989) Multiple isoforms of human microtubule-associated protein tau: sequences and localization in neurofibrillary tangles of Alzheimer's disease. Neuron 3:519-526.

Goedert M, Crowther RA, Spillantini MG (1998) Tau mutations cause frontotemporal dementia. Neuron 21:955-958.

Goedert M, Jakes R, Crowther RA (1999) Effects of frontotemporal dementia FTDP-17 mutations on heparin-induced assembly of tau filaments. FEBS Lett 450:306-311.

Goode BL, Chau M, Denis PE, Feinstein SC (2000) Structural and functional differences between 3-repeat and 4-repeat tau isoforms: implications for normal tau function and onset of neurodegenerative disease. J Biol Chem 275:38182-38189.

Götz J, Probst A, Spillantini MG, Schäfer T, Jakes R, Bürki K, Goedert M (1995) Somatodendritic localization and hyperphosphorylation of tau protein in transgenic mice expressing the longest human brain tau isoform. EMBO J 14:1304-1313.

Götz J, Chen F, van Dorpe J, Nitsch RM (2001) Formation of neurofibrillary tangles in $\mathrm{P} 301 \mathrm{~L}$ tau transgenic mice induced by $\mathrm{A} \beta_{42}$ fibrils. Science 293:1491-1495.

Gunawardena S, Goldstein LSB (2001) Disruption of axonal transport and neuronal viability by amyloid precursor protein mutations in Drosophila. Neuron 32:389-401.

Gustke N, Trinczek B, Biernat J, Mandelkow EM, Mandelkow E (1994) Domains of tau protein and interactions with microtubules. Biochemistry 33:9511-9522.

Hasegawa M, Smith MJ, Goedert M (1998) Tau proteins with FTDP-17 mutations have a reduced ability to promote microtubule assembly. FEBS Lett 437:207-210.

Hirokawa N, Terada S, Funakoshi T, Takeda S (1997) Slow axonal transport: the subunit model. Trends Cell Biol 7:384-388.

Hong M, Zhukareva V, Vogelsberg-Ragaglia V, Wszolek Z, Reed L, Miller BI, Geschwind DH, Bird TD, McKeel D, Goate A, Morris JC, Wilhelmsen KC, Schellenberg GD, Trojanowski JQ, Lee VM-Y (1998) Mutation-specific functional impairments in distinct tau isoforms of hereditary FTDP-17. Science 282:1914-1917.

Hutton M, Lendon CL, Rizzu P, Baker M, Froelich S, Houlden H, Pickering-Brown S, Chakraverty S, Issacs A, Grover A, Hackett J, Adamson J, Lincoln S, Dickson D, Davies P, Petersen RC, Stevens M, de Graaf E, Wauters E, van Baren J et al. (1998) Association of missense and $5^{\prime}$-splice mutations in tau with the inherited dementia FTDP-17. Nature 393:702-705.

Ishihara T, Hong M, Zhang B, Nakagawa Y, Lee MK, Trojanowski JQ, Lee VM-Y (1999) Age-dependent emergence and progression of a tauopathy in transgenic mice overexpressing the shortest human tau isoform. Neuron 24:751-762.

Lee G, Rook SL (1992) Expression of tau protein in non-neuronal cells: microtubule binding and stabilization. J Cell Biol 102:227-237.

Lee VM-Y, Goedert M, Trojanowski J (2001) Neurodegenerative tauopathies. Annu Rev Neurosci 24:1121-1159.

Léger J, Brandt R, Lee G (1994) Identification of tau protein regions required for process formation in PC12 cells. J Cell Sci 107:3403-3412.

Maas T, Eidenmuller J, Brandt R (2000) Interaction of tau with the neural membrane cortex is regulated by phosphorylation at sites that are modified in paired helical filaments. J Biol Chem 275:15733-15740.

Mercken M, Fischer I, Kosik KS, Nixon RA (1995) Three distinct axonal transport rates for tau, tubulin, and other microtubule-associated proteins: evidence for dynamic interactions of tau with microtubules in vivo. J Neurosci 15:8259-8267.

Nacharaju P, Lewis J, Easson C, Yen S, Hackett J, Hutton M, Yen S-H (1999) Accelerated filament formation of tau protein with specific FTDP-17 missense mutations. FEBS Lett 447:195-199.
Nikolic MH, Dudek YT, Kwon YT, Ramos YFM, Tsai LH (1996) The $\mathrm{cdk} 5 / \mathrm{p} 35$ kinase is essential for neurite outgrowth during neuronal differentiation. Genes Dev 10:816-825.

Nixon RA (1998) The slow axonal transport of cytoskeletal proteins. Curr Opin Cell Biol 10:87-92.

Poorkaj P, Bird TD, Wijsman E, Nemens E, Garruto RM, Anderson L, Andreadis A, Wiederholt WC, Raskind M, Schellenberg GD (1998) Tau is a candidate gene for chromosome 17 frontotemporal dementia. Ann Neurol 43:815-825.

Rizzu P, van Swieten JC, Joosse M, Hasegawa M, Stevens M, Tibben A Niermeijer MF, Hillebrand M, Ravid R, Oostra BA, Goedert M, van Duijin CM, Heutink P (1999) High prevalence of mutations in the microtubule-associated protein tau in a population study of frontotemporal dementia in The Netherlands. Am J Hum Genet 64:414-421.

Sambrook J, Fritsch EF, Maniatis T (1989) Molecular cloning: a laboratory manual. Cold Spring Harbor, NY: Cold Spring Harbor Laboratory.

Spillantini MG, Murrell JR, Goedert M, Farlow MR, Klug A, Ghetti B (1998) Mutations in the tau gene in familial multiple system tauopathy with presenile dementia. Proc Natl Acad Sci USA 95:7737-7741.

Stamer K, Vogel R, Thies E, Mandelkow E, Mandelkow EM (2002) Tau blocks traffic of organelles, neurofilaments, and APP vesicles in neurons and enhances oxidative stress. J Cell Biol 156:1051-1063.

Tashiro T, Sun X, Tsuda M, Komiya Y (1996) Differential axonal transport of soluble and insoluble tau in the rat sciatic nerve. $J$ Neurochem 67:1566-1574.

Tesseur I, van Dorpe J, Bruynseels K, Bronfman F, Sciot R, van Lommel A, van Leuven F (2000) Prominent axonopathy and disruption of axonal transport in transgenic mice expressing human apolipoprotein E4 in neurons of brain and spinal cord. Am J Pathol 157:1495-1510.

Torroja L, Chu H, Kotovsky I, White K (1999) Neuronal overexpression of APPL, the Drosophila homologue of the amyloid precursor protein (APP), disrupts axonal transport. Curr Biol 9:489-492.

Trinczek B, Ebneth A, Mandelkow EM, Mandelkow E (1999) Tau regulates the attachment/detachment but not the speed of motors in microtubule-dependent transport of single vesicles and organelles. J Cell Sci 112:2355-2367.

Tytell M, Brady ST, Lasek RJ (1984) Axonal transport of a subclass of tau proteins: evidence for the regional differentiation of microtubules in neurons. Proc Natl Acad Sci USA 81:1570-1574.

Utton MA, Gibb GM, Burdett IDJ, Anderton BH, Vandecandelaere A (2001) Functional differences of tau isoforms containing 3 or 4 $\mathrm{C}$-terminal repeat regions and the influence of oxidative stress. $\mathrm{J}$ Biol Chem 276:34288-34297.

van Slegtenhorst M, Lewis J, Hutton M (2000) The molecular genetics of tauopathies. Exp Gerontol 35:461-471.

Varini L, Hasegawa M, Spillantini MG, Smith MJ, Murrell JR, Ghetti B, Klug A, Goedert M, Varini G (1999) Structure of tau exon 10 splicing regulatory element RNA and destabilization by mutation of frontotemporal dementia and parkinsonism linked to chromosome 17. Proc Natl Acad Sci USA 96:8229-8234.

Vogelsberg-Ragaglia V, Bruce J, Richter-Landsberg C, Zhang B, Hong M, Trojanowski JQ, Lee VM-Y (2000) Distinct FTDP-17 missense mutations in tau produce tau aggregates and other pathological phenotypes in transfected CHO cells. Mol Biol Cell 11:4093-4104.

von Bergen M, Barghorn S, Li L, Marx A, Biernat J, Mandelkow EM, Mandelkow E (2001) Mutations of tau protein in frontotemporal dementia promote aggregation of paired helical filaments by enhancing local $\beta$-structure. J Biol Chem 276:48165-48174.

Williamson TL, Cleveland DW (1999) Slowing of axonal transport is a very early event in the toxicity of ALS-linked Sod1 mutations to motor neurons. Nat Neurosci 2:50-56.

Winckler B, Mellman I (1999) Neuronal polarity: controlling the sorting and diffusion of membrane components. Neuron 23:637-640.

Xia ZG, Dudek H, Miranti CK, Greenberg ME (1996) Calcium influx via the NMDA receptor induces immediate-early gene transcription by a MAP kinase/ERK-dependent mechanism. J Neurosci 16:5425-5436.

Yen S, Easson C, Nacharaju P, Hutton M, Yen S-H (1999) FTDP-17 tau mutations decrease the susceptibility of tau to calpain I digestion. FEBS Lett 461:91-95.

Yoon M, Moir RD, Prahlad V, Goldman RD (1998) Motile properties of vimentin intermediate filament networks in living cells. J Cell Biol 143:147-157.

Zhang P, Tu P, Abrabian F, Trojanowski JQ, Lee VM (1997) Neurofilaments and orthograde transport are reduced in ventral root axons of transgenic mice that express human SOD1 with a G93A mutation. J Cell Biol 139:1307-1315.

Zhao C, Takita J, Tanaka Y, Setou M, Nakagawa T, Takeda S, Takei Y, Saito M, Tsuji S, Hayashi Y, Hirokawa N (2001) Charcot-MarieTooth disease type $2 \mathrm{~A}$ caused by mutation in a microtubule motor KIF1B $\beta$. Cell 105:587-597. 